\title{
Flutuação populacional de Anastrepha fraterculus (Wiedemann, 1830) (Diptera, Tephritidae) na Região Oeste de Santa Catarina, Brasil ${ }^{1}$
}

\author{
Flávio Roberto Mello Garcia ${ }^{2,3}$ \\ Jocélia Vargas Campos ${ }^{2}$ \\ Elio Corseuil ${ }^{3}$
}

\begin{abstract}
Population fluctuation of Anastrepha fraterculus (Wiedemann, 1830) (Diptera, Tephritidae) in the Western Region of Santa Catarina State, Brazil. Fruit flies are the major pests in fruit orchards and require a frequent insecticide aplication control, which increases production cost and chemical residues in fruits. Adults of Anastrepha fraterculus were sampled from twelve peach, plum, orange, tangerine and acid lime orchards in four counties in the Western Region of Santa Catarina. Modified McPhail plastic traps, baited with glucose 10\%, were used to collect the flies from October 1998 to September 2000. Trap monitoring, bait replacement and fruit flies sorting by species and sex were done weekly. A total of 4,164 specimens of A. fraterculus was collected and highest population was registered in the county of Chapecó (64,8\% of all sampled flies). Adults were collected all year long, with the highest population peaks occurring from December and January, although the fluctuation was different for each fruit species due to their particular phenology and in different years. Positive correlation among temperature, atmospheric humidity and population levels of adults of A. fraterculus was observed. According to the degree days obtained for each year, 4851.9, 4632.9 and 4983.7, respectively in 1998, 1999 and 2000, it was established that $A$. fraterculus could present an average of 11.2 generations a year.
\end{abstract}

KEYwords. Insect monitoring; orchard pests; south american fruit fly

\section{INTRODUÇÃO}

O estado de Santa Catarina sobressai-se no cenário nacional como um grande produtor de frutas, principalmente de clima temperado. O cultivo de citros é expressivo no oeste do estado. A incidência de moscas-das-frutas é um fator preocupante todos os anos, causando aumento nos custos de produção em razão das freqüentes aplicações de inseticidas para seu controle e das perdas na produção (Nora et al. 2000).

Segundo Malavasi et al. (2000), Anastrepha fraterculus (Wiedemann, 1830) é uma praga primária da maior importância na Argentina, Uruguai, e nos Estados do sul e sudeste do Brasil, sendo que, nestes locais, se concentram as medidas de controle e as maiores perdas. Das 70 espécies de moscas-das- frutas assinaladas para Santa Catarina (GARCIA et al. 2002), A. fraterculus é a espécie de maior distribuição e abundância nas regiões produtoras catarinenses (NorA et al. 2000).

Anastrepha fraterculus é uma espécie multivoltina, apresentando, no mínimo, seis gerações anuais o que possibilita sua presença durante todos os meses do ano (MAchado et al. 1995; Salles 1995). Além disso, esta espécie é polífaga, sendo conhecidos 67 hospedeiros de 18 famílias (ZucCHI 2000b).

Bleicher et al. $(1978,1982)$ e Hickel \& Ducroquet (1993) constataram que o pico de ocorrência de $A$. fraterculus foi em dezembro em pomares de ameixeira e pessegueiro no município de Videira, Santa Catarina. Bleicher \& Bleicher (1980) e BLEICHER et al. (1982) observaram que a maior incidência de $A$. fraterculus se deu de janeiro a fevereiro em pomares de macieira

1. Parte de tese de doutorado do primeiro autor aprovada na PUCRS.

2. Universidade Comunitária Regional de Chapecó, Centro de Ciências Agro-ambientais e de Alimentos, Laboratório de Entomologia. Caixa Postal 747, 89809-000 Chapecó-SC, Brasil. Endereço eletrônico: flaviog@unochapeco.rct-sc.br

3. Pontifícia Universidade Católica do Rio Grande do Sul. Faculdade de Biociências, Laboratório de Entomologia. Av. Ipiranga 6681, 90619-900 Porto Alegre-RS, Brasil. 
no município de Fraiburgo, Santa Catarina.

O presente trabalho teve como objetivo estudar a flutuação populacional de A. fraterculus na Região Oeste de Santa Catarina.

\section{MATERIAL E MÉTODOS}

Para coleta de adultos foram instaladas armadilhas caçamoscas do tipo McPhail, com $200 \mathrm{ml}$ de solução aquosa de glicose invertida a 10\% (GARCIA et al. 1999), colocadas a uma altura aproximada de 1,5 m, na copa das árvores. Foram efetuadas coletas em doze pomares de quatro municípios: Chapecó (27ำ 09'S; 52 61'W, altitude de $670 \mathrm{~m}$ ) em pomar de laranjeiras (Citrus sinensis cv. Valência), limoeiros (Citrus aurantifolia cv. Tahiti), pessegueiros (Prunus persica cv. Marli) e tangerineiras (Citrus reticulata cv. Montenegrina); Cunha Porã (26 89'S, 53 16'W, altitude de $456 \mathrm{~m}$ ) em pomar de pessegueiros e tangerineiras; em São Carlos (27º 07’ S; 53 00’W, altitude 264 m) em pomar de ameixeiras (Prunus domestica cv. Santa Rosa), laranjeiras, pessegueiros e tangerineiras e Xanxerê ( $26^{\circ} 87^{\prime} \mathrm{S}$; 52 $2^{\circ} 40^{\prime} \mathrm{W}$, altitude de $800 \mathrm{~m}$ ) em pomar de pessegueiros e tangerineiras.

Foram utilizados quatro frascos por pomar, perfazendo um total de 48, distribuídos nas bordas dos pomares e distanciados cerca de 48 m (Martinez \& Godoy 1987). Durante o período de outubro de 1998 a setembro de 2000 foram realizadas visitas semanais aos pomares para troca da solução atrativa e coleta dos tefritídeos, colocando-os em frascos etiquetados, contendo álcool 70\%, para posterior sexagem, contagem e identificação em laboratório.

A identificação das espécies de Anastrepha foi baseada no acúleo. Para tanto, as moscas foram colocadas na posição ventral em uma lâmina sob microscópio estereoscópico e, com auxílio de dois estiletes, foi extrovertido o acúleo. O exame do acúleo foi feito sob aumento de 40 ou 100 vezes, neste caso, foi colocada uma gota de glicerina. Posteriormente, foi usada a chave pictórica de STEYSKAL (1977) para espécies de Anastrepha e a dicotômica de Zucchi (2000a) para as espécies ocorrentes no Brasil.

Foram feitas anotações sobre a fenologia do vegetal. Estas informações serviram para comparar com as etapas da cultura e a flutuação populacional dos tefritídeos.

Os dados provenientes das coletas de adultos de $A$. fraterculus foram utilizados para estudo de flutuação populacional e análise de regressão múltipla com os fatores meteorológicos através do programa Microstat para o número de moscas coletadas mensalmente.

Os dados meteorológicos do município de Chapecó, representados por temperatura máxima, temperatura média, temperatura mínima e umidade relativa do ar, para cada semana de coleta, calculados a partir dos dados da Estação Experimental do EPAGRI/CLIMERH. Estimou-se o número de gerações de A. frateculus em Chapecó, utilizando-se os dados de temperatura mínima dos anos de 1998, 1999 e 2000 e considerando a temperatura-base de $10,72{ }^{\circ} \mathrm{C}$ e a necessidade térmica de 430,58 Graus-dia para o desenvolvimento do ciclo evolutivo determinado por MACHADO et al. (1995).

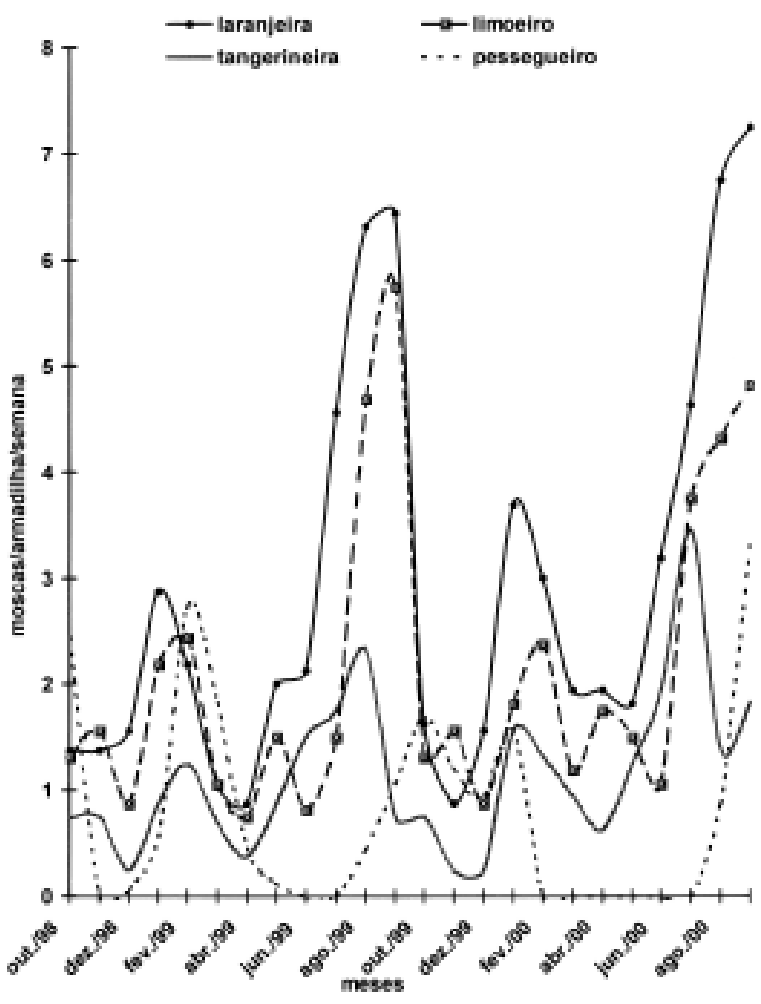

Fig. 1. Flutuação populacional de Anastrepha fraterculus (Wied., 1830) (Diptera, Tephritidae) coletadas com frascos McPhail em quatro pomares do município de Chapecó, SC, de outubro de 1998 a setembro de 2000.

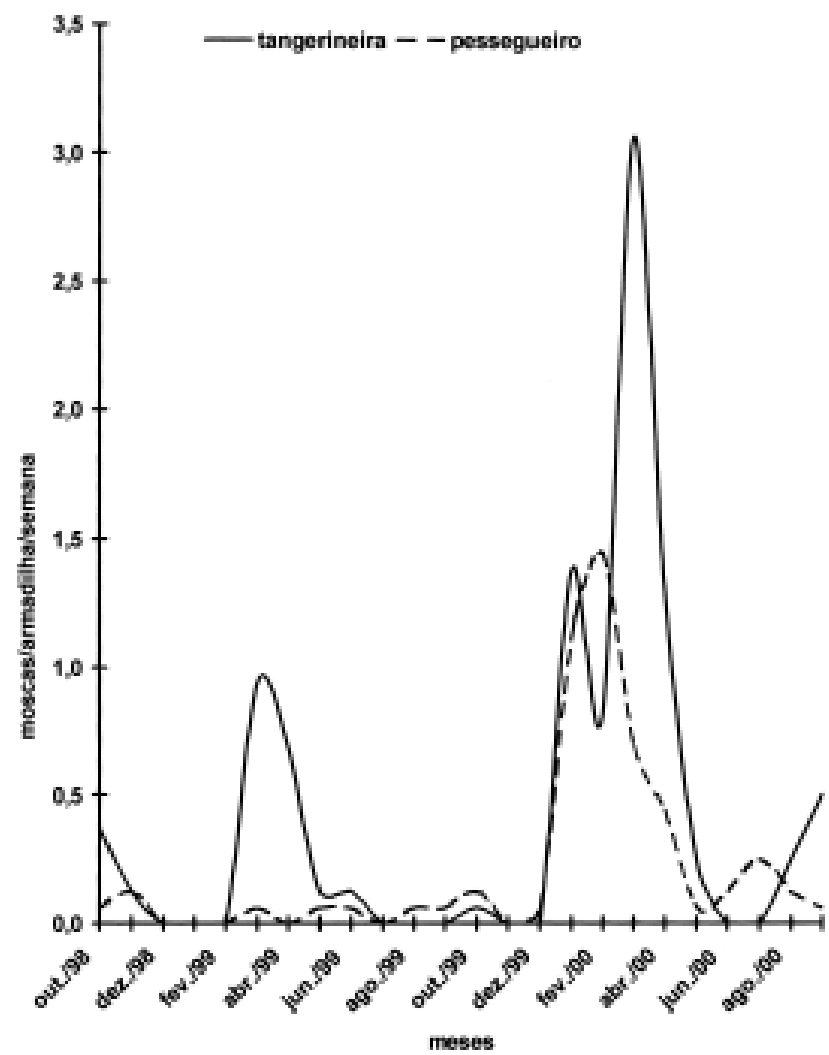

Fig. 2. Flutuação populacional de Anastrepha fraterculus (Wied., 1830) (Diptera, Tephritidae) coletadas com frascos McPhail em dois pomares do município de Cunha Porã, SC, de outubro de 1998 a setembro de 2000. 


\section{RESULTADOS E DISCUSSÃO}

Coletou-se um total de 4.164 espécimes de $A$. fraterculus, sendo que 64,8 \% foram obtidos em Chapecó, 21,3\% em São Carlos, 8,1\% em Xanxerê e apenas 5,8\% em Cunha Porã.

Os picos populacionais de $A$. fraterculus foram em novembro de 1998, março de 1999 e setembro de 2000, no pomar de pessegueiro no município de Chapecó. No pomar de laranjeira, o pico populacional, nos dois anos, foi em janeiro e de agosto a setembro; no de limoeiro foi em março e setembro, em ambos os anos e no de tangerineira, foi em setembro de 1999 e julho de 2000 (Fig. 1).

Em Cunha Porã, as maiores ocorrências de $A$. fraterculus foram em março e abril no pomar de tangerineira, com um pico isolado em janeiro de 2000. Em pessegueiro, ocorreu um único pico em fevereiro e março de 2000 (Fig. 2).

No município de São Carlos, no pomar de ameixeira obtevese picos em outubro de 1998 e dezembro de 1999, concomitante com a frutificação. A maior ocorrência em marçolabril deve-se possivelmente à maior frutificação de araçá (Psidium cattleianum Sabine), goiaba (Psidium guajava L.) e setecapotes (Britoa guazumaefolia (Camb.)) na região, haja visto que não havia ameixas nos pomares. Em pomar de pessegueiro, na mesma cidade, ocorreram picos em dezembro de 1998 e 1999, além de junho de 1999. No pomar de laranjeira, os picos, nos dois anos, foram em fevereiro e junho, enquanto que em tangerineira, ocorreram em junho e julho (Fig. 3).

Os picos de $A$. fraterculus em pessegueiro no município de Xanxerê, foram em janeiro nos dois anos e em março de 1999. No pomar de tangerineira, em janeiro nos dois anos, e um outro em março de 2000 (Fig. 4).

Nos quatro municípios, a maior incidência de $A$. fraterculus em pessegueiro ocorreu nos meses de dezembro e janeiro, concomitante com a frutificação, corroborando os resultados obtidos em pomares de pessegueiro em diversas regiões do Brasil: BLEICHER et. al. (1978, 1982), FEHN (1982), LORENZATO et al. (1986), Matioli et al. (1988), Salles (1995, 1997, 2000), SALles \& Kovaleski (1990), Hickel \& Ducroquet (1993), Garcia \& Corseuil (1998a, b) e Nora et al. (2000). Além disso, os picos dessa espécie em pessegueiro assemelharam-se aos ocorrentes em pomares de citros (Puzzi \& Orlando 1965); macieira (Bleicher \& BleICHER 1980; Bleicher et al. 1982; LORENZATO \& CHOUENe 1985; OrTH et al. 1986), pereira (NorA 2001); e quivi (HiCKel \& SснUск 1993), em diferentes localidades do Brasil.

Os picos populacionais das moscas-das-frutas em pomares cítricos variaram de um ano para outro e entre pomares em um mesmo ano, não apresentando picos bem definidos, o que também foi detectado por RaGa et. al (1996) em pomar de citros no município de Presidente Prudente, SP.

As médias dos fatores climáticos mensais e o número de moscas/frasco encontra-se na Tabela I. Obteve-se significância estatística $(\mathrm{P}<0,05)$ para regressão entre os fatores climáticos e os níveis populacionais de $A$. fraterculus em três pomares de Chapecó, não se obtendo apenas para o pomar de pessegueiro, possivelmente pelo baixo número de espécimes coletados. A temperatura máxima atuou de forma positiva enquanto a

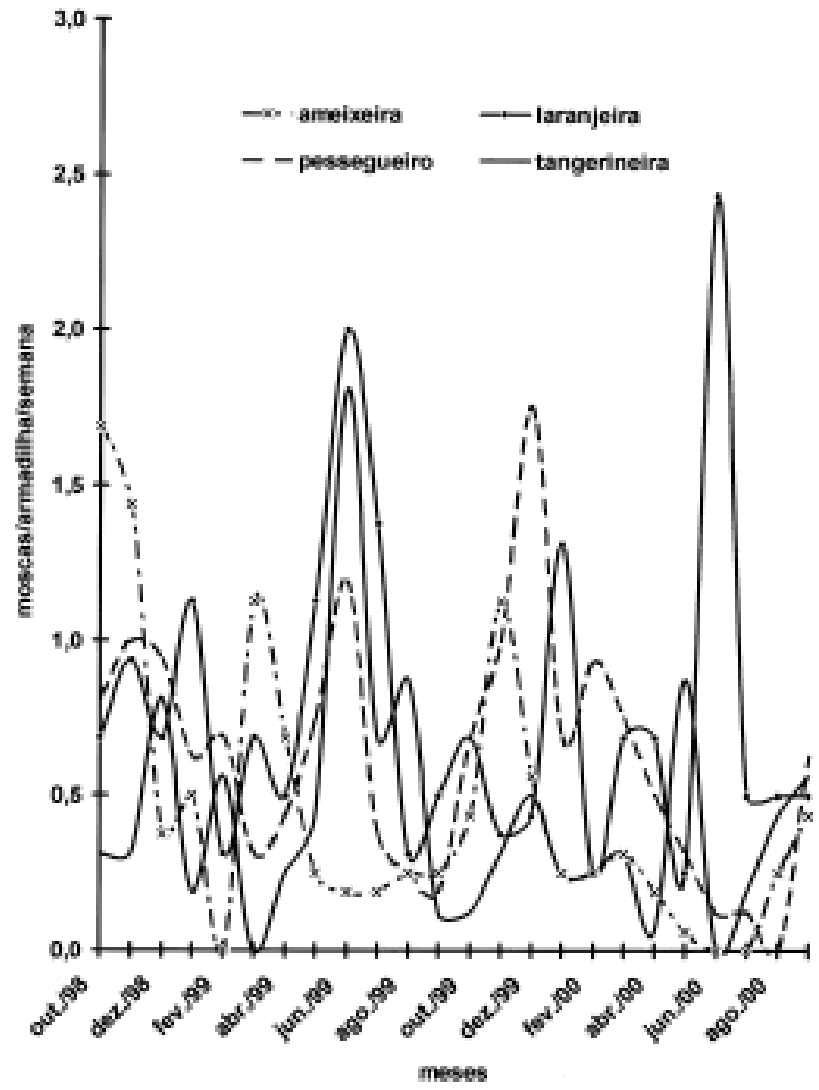

Fig. 3. Flutuação populacional de Anastrepha fraterculus (Wied., 1830) (Diptera, Tephritidae) coletadas com frascos McPhail em quatro pomares do município de São Carlos, SC, de outubro de 1998 a setembro de 2000.

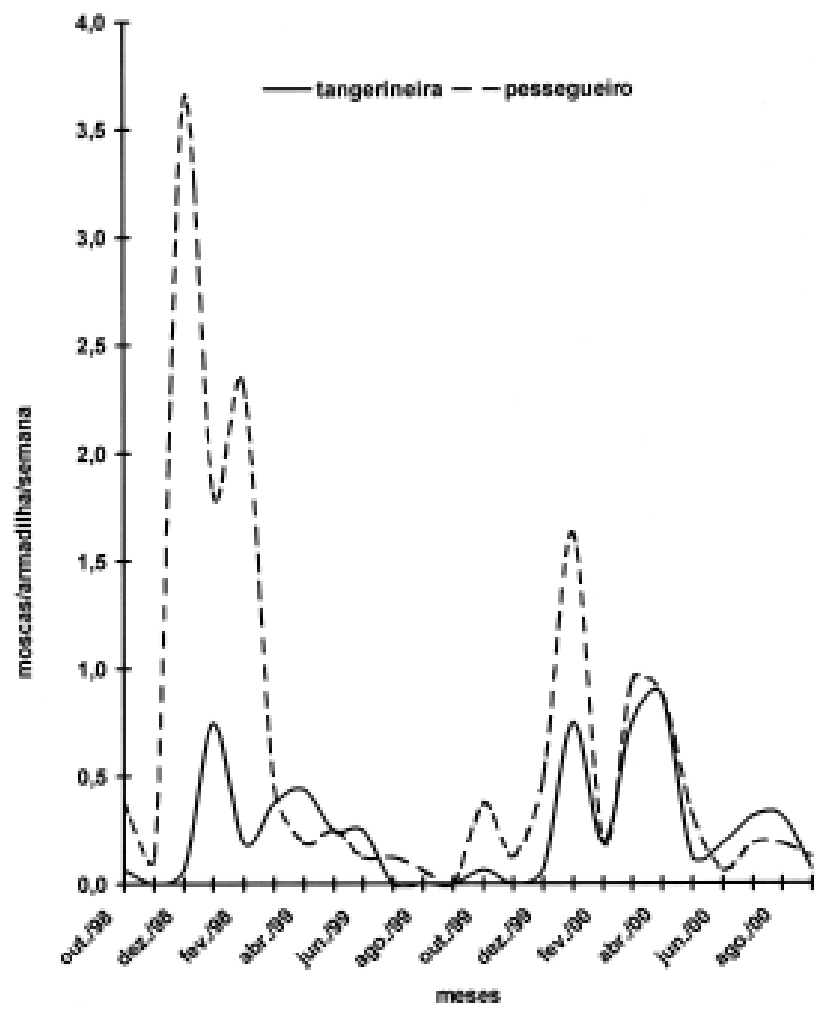

Fig. 4. Flutuação populacional de Anastrepha fraterculus (Wied., 1830) (Diptera, Tephritidae) coletadas com frascos McPhail em dois pomares do município de Xanxerê, SC, de outubro de 1998 a setembro de 2000. 
Tabela I. Dados mensais sobre capturas de adultos de $A$. fraterculus, temperaturas $\left({ }^{\circ} \mathrm{C}\right)$ máximas (TMA), médias (TME) e mínimas (TMI) e umidade relativa do ar (UR\%) em Chapecó, SC, no período de outubro de1998 a setembro de 2000

\begin{tabular}{|c|c|c|c|c|c|c|c|}
\hline \multirow[t]{2}{*}{ Meses } & \multicolumn{4}{|c|}{ Dados meteorológicos } & \multicolumn{3}{|c|}{$\begin{array}{l}\text { Média do } n^{\circ} \text { de espécimes } \\
\text { de } A \text {. fraturculus / frasco }\end{array}$} \\
\hline & UR & TMA & TMI & TME & laranja & limão & tangerina \\
\hline out./98 & 74,22 & 23,34 & 16,32 & 19,52 & 5,50 & 5,250 & 3,00 \\
\hline nov./98 & 61,27 & 26,25 & 18,19 & 21,89 & 5,50 & 6,250 & 3,00 \\
\hline dez./98 & 66,84 & 25,89 & 19,04 & 22,23 & 6,25 & 3,500 & 1,00 \\
\hline jan./99 & 71,28 & 27,58 & 19,81 & 23,42 & 11,5 & 8,750 & 3,50 \\
\hline fev./99 & 76,38 & 26,77 & 19,95 & 23,06 & 8,75 & 9,750 & 5,00 \\
\hline mar./99 & 69,70 & 27,67 & 20,30 & 23,55 & 4,25 & 4,250 & 2,75 \\
\hline abr./99 & 77,16 & 22,26 & 15,58 & 18,54 & 3,50 & 3,000 & 1,50 \\
\hline mai./99 & 76,12 & 18,53 & 11,54 & 14,73 & 8,00 & 6,00 & 3,50 \\
\hline jun./99 & 80,37 & 16,73 & 10,42 & 13,39 & 8,50 & 3,25 & 6,00 \\
\hline jul./99 & 80,58 & 17,15 & 10,72 & 13,70 & 18,25 & 6,00 & 7,00 \\
\hline ago./99 & 58,92 & 20,92 & 12,39 & 16,19 & 25,25 & 18,75 & 9,25 \\
\hline set./99 & 60,56 & 22,70 & 14,67 & 18,65 & 25,75 & 23,00 & 3,00 \\
\hline out./99 & 69,99 & 21,74 & 14,94 & 17,95 & 6,50 & 5,25 & 3,00 \\
\hline nov./99 & 59,13 & 24,35 & 16,38 & 20,22 & 3,500 & 6,25 & 1,00 \\
\hline dez./99 & 64,33 & 27,05 & 19,72 & 23,09 & 6,25 & 3,50 & 1,00 \\
\hline jan./00 & 68,90 & 27,85 & 20,49 & 23,81 & 14,75 & 7,25 & 6,25 \\
\hline fev./00 & 73,55 & 26,79 & 19,80 & 23,01 & 12,00 & 9,50 & 5,25 \\
\hline mar./00 & 73,00 & 25,71 & 18,62 & 21,90 & 7,75 & 4,75 & 3,75 \\
\hline abr./00 & 69,82 & 23,95 & 16,65 & 19,92 & 7,75 & 7,00 & 2,50 \\
\hline mai./00 & 75,46 & 18,61 & 11,63 & 14,69 & 7,25 & 6,00 & 5,00 \\
\hline jun./00 & 75,42 & 19,45 & 13,82 & 16,42 & 12,75 & 4,250 & 7,75 \\
\hline jul./00 & 69,43 & 15,31 & $\begin{array}{r}7,02 \\
7,55\end{array}$ & 11,46 & 18,50 & 15,00 & 13,75 \\
\hline ago./00 & 63,64 & 20,39 & 12,38 & 16,12 & 27,00 & 17,25 & 5,50 \\
\hline set./00 & 64,32 & 22,06 & 13,88 & 17,02 & 29,00 & 19,25 & 7,25 \\
\hline
\end{tabular}

temperatura mínima, de forma negativa sobre a população de A. fraterculus nos três pomares. A temperatura média e a umidade relativa atuaram no crescimento positivo da população apenas no pomar de tangerineira (Tabela II).

A influência da temperatura sobre populações de $A$. fraterculus foi constatada, também, por BLEICHER \& BLEICHER (1980), FeHn (1981), LORENZATO \& ChOUENE (1985), SuPLicy FiLHO et al. (1987), SAlLES (1993a, 1995), MACHADO et al. (1995), SALLES et al. (1995), RaGa et al. (1996), Garcia \& Corseuil (1998c) e TAUFER et al. (2000).
Não se conhece o efeito da umidade relativa do ar sobre a biologia e comportamento desta espécie. Presume-se que esta variável climática possa ter influência apenas sobre os adultos (SAlLES 1995, 2000). Todavia, o efeito da umidade relativa do ar sobre tefritídeos foi mencionada por ZAHLER (1990), SALLES et al. (1995), Aguiar-MEnEZes \& MenEzes (1996), Raga et al. (1996) e GARCIA \& CORSEUIL (1998c).

Obteve-se em Chapecó 4851,90 Graus-dia no ano de 1998; de 4632,90 no ano de 1999 e de 4983,70 em 2000, estimando-se que $A$. fraterculus tem condições de desenvolver, pelo menos,

Tabela II. Coeficientes de regressão $(\mathrm{Cr})$ e coeficientes de determinação parciais $\left(\mathrm{r}^{2}\right)$ obtidos através de regressão múltipla, para o número de espécimes de Anastrepha fraterculus capturados com armadilhas tipo McPhail em três pomares do município de Chapecó, SC, em função dos fatores climáticos, no período de outubro de 1998 a setembro de 2000 .

\begin{tabular}{|c|c|c|c|c|c|c|}
\hline \multirow{3}{*}{ Fatores } & \multicolumn{6}{|c|}{ Anastrepha fraterculus } \\
\hline & \multicolumn{2}{|c|}{ laranja } & \multicolumn{2}{|c|}{ limão } & \multicolumn{2}{|c|}{ tangerina } \\
\hline & $\mathrm{Cr}$ & $r^{2}$ & $\mathrm{Cr}$ & $\mathrm{r}^{2}$ & $\mathrm{Cr}$ & $r^{2}$ \\
\hline temperatura máxima & 7,59 & 0,1800 & 5,84 & 0,1700 & 1,44 & 0,3900 \\
\hline temperatura média & $-7,06$ & 0,0390 & $-0,65$ & 0,0009 & 0,09 & 0,9770 \\
\hline temperatura mínima & $-1,54$ & 0,0340 & $-5,71$ & 0,1078 & $-1,99$ & 0,0005 \\
\hline umidade relativa & $-0,15$ & 0,0070 & $-0,47$ & 0,0020 & 0,13 & 0,0380 \\
\hline constante & 670 & & $-20,19$ & & $-8,35$ & \\
\hline Significância & \multicolumn{2}{|c|}{0,023} & \multicolumn{2}{|c|}{0,00002} & \multicolumn{2}{|c|}{0,0355} \\
\hline
\end{tabular}


11,27; 10,75 e 11,57 gerações anuais, respectivamente, considerando exclusivamente a temperatura. Em Pelotas, RS, MACHADO et al. (1995) estimaram a possibilidade de que essa espécie poderia desenvolver, no mínimo, seis gerações anuais. Os dados obtidos em Chapecó indicam que $A$. fraterculus não tem diapausa na região, o que foi concluído também por SALLES (1993b) e MACHADO et al. (1995) para a região de Pelotas, RS. Segundo Nora (2001) a grande diversidade de plantas hospedeiras nativas e cultivadas em Santa Catarina, com diferentes épocas de frutificação, facilita a reprodução sucessiva de $A$. fraterculus durante o ano todo.

\section{CONCLUSÕES}

A partir dos resultados obtidos pode-se concluir que: 1) o pico populacional de $A$. fraterculus é variável a cada ano e em diferentes culturas e localidades; 2) obteve-se correlação significativa entre a temperatura e umidade relativa do ar e os níveis populacionais de $A$. fraterculus, 3 ) os adultos de $A$. fraterculus ocorrem em todas as estações do ano.

Agradecimentos. Desejamos externar nossos sinceros agradecimentos à Geógrafa Vera Lúcia da Silva do setor de Banco de Dados da EPAGRI/ CLIMERH, pela cedência dos dados meteorológicos.

\section{REFERÊNCIAS}

Aguiar-Menezes, E. L. \& E. B. Menezes. 1996. Flutuação populacional das moscas das frutas e a sua relação com a disponibilidade hospedeira em Itaguaí, RJ. Anais da Sociedade Entomológica do Brasil 25(2): 223-232.

Bleicher, J.; D. N. Gassen; L. G. Ribeiro; H. Tanaka, \& A. I. Orth. 1982 Moscas-das-frutas em macieira e pessegueiro . Florianópolis, EMPASC, 28 p.

Bleicher, E. \& J. Bleicher. 1980. Flutuação populacional da mosca da fruta Anastrepha fratercula em macieira no município de Fraiburgo/SC. Florianópolis, Secretaria da Agricultura e Abastecimento, $8 \mathrm{p}$.

Bleicher, E.; A-L. Schroeder \& J. Bleicher. 1978. Flutuação da “moscas das frutas” (Anastrepha fratercula Wied., 1830) no município de Videira. Florianópolis, Secretaria da Agricultura e Abastecimento, 9p.

Fenn, L. M. 1981. Coleta e reconhecimento de moscas das frutas na Região Metropolitana de Curitiba e Irati, Paraná, Brasil. Anais da Sociedade Entomológica do Brasil 10(2): 199-208.

Fenn, L. M. 1982. Influência dos fatores meteorológicos na flutuação e dinâmica de população de Anastrepha spp. Pesquisa Agropecuária Brasileira 17(4): 533-544.

Garcia, F. R. M. \& E. Corseuil. 1998a. Flutuação populacional de Anastrepha fraterculus (Wiedemann) e Ceratitis capitata (Wiedemann) (Diptera, Tephritidae) em pomares de pessegueiro em Porto Alegre, Rio Grande do Sul. Revista Brasileira de Zoologia 15(1): 153-158.

Garcia, F. R. M. \& E. Corseuil. 1998b. Análise faunística de moscas-dasfrutas (Diptera, Tephritidae) em pomares de pessegueiro em Porto Alegre, Rio Grande do Sul. Revista Brasileira de Zoologia 15(4): 1111-1117.

Garcia, F. R. M. \& E. CorseuIL. 1998c. Influência de fatores climáticos sobre moscas-das-frutas (Diptera, Tephritidae) em pomares de pessegueiro em Porto Alegre, Rio Grande do Sul. Revista da Faculdade de Zootecnia, Veterinária e Agronomia 5/6(1): 7175.
Garcia, F. R. M; J. V. CAmpos \& E. CorseuIL. 1999. Avaliação de atrativos na captura de adultos de Anastrepha fraterculus (Wied, 1830) (Diptera, Tephritidae) Biociências 7(1): 43-50.

Garcia, F. R. M; J. V. Campos \& E. Corseuil. 2002. Lista documentada das moscas-das-frutas (Diptera, Tephritidae) de Santa Catarina, Brasil. Biociências 10(1): 139-148.

Hickel, E. R. \& J-P. H. J. Ducroquet. 1993. Flutuação populacional de espécies de Anastrepha sp. (Diptera: Tephritidae) relacionadas com a fenologia de frutificação do pêssego e ameixa em Santa Catarina. Anais da Sociedade Entomológica do Brasil 22(3): 591-596.

Hickel, E. R. \& E. Schuck. 1993. Ocorrência da mosca-das-frutas, Anastrepha fraterculus (Diptera: Tephritidae) em frutos de Quivi. Pesquisa Agropecuária Brasileira 28(11): 1345-1347.

LoRENZATo, D. \& E. C. Chouene. 1985. Flutuação populacional, efeitos da temperatura e manejo de moscas-das-frutas do gênero Anastrepha em pomares de macieira (Malus domestica Borck) no município de Farroupilha, RS. Agronomia Sulriograndense 21(2): 297-319.

Lorenzato, D.; E. O. Grelmann \& E. C. Chouene. 1986. Monitoramento e manejo de moscas-das-frutas do gênero Anastrepha em pomares de macieiras, pereiras e pessegueiros no município de Veranópolis - RS. Agronomia Sulriograndense 22(1): 105-134.

Machado, A. E.; L. A. B. Salles \& A. E. Loeck. 1995. Exigências térmicas de Anastrepha fraterculus (Wied.,) e estimativa do número de gerações anuais em Pelotas, RS. Anais da Sociedade Entomológica do Brasil 24(3): 573-578.

Malavasi, A.; R. A. Zucchi \& R. L. Sugayama. 2000. Biogeografia, p. 9398. In: A. Malavasi \& R. A. Zucchi (edit.). Moscas-das-frutas de importância econômica no Brasil: conhecimento básico e aplicado. Ribeirão Preto, Holos Editora, 327 p.

Martinez, N. B. De \& F. J. Godoy. 1987. Distanciamento entre trampas McPhail en la captura de adultos de Anastrepha sp. (Diptera: Tephritidae). Agronomia Tropical 37: 121-124.

Matioli, J. C.; M. M. Rossi \& V. H. Bueno. 1988. Suscetibilidade de cultivares de pêssego às moscas das frutas (Diptera: Tephritidae) na região de Caldas - MG. Observações preliminares. Anais da Sociedade Entomológica do Brasil. 17(suplemento): 75-85.

Nora, I. 2001. Mosca-das-frutas (Anastrepha fraterculus) (Wiedemann, 1830) (Diptera: Tephritidae), p. 284-298. In: EPAGRI (edit.) Nashi, a pêra japonesa. Florianópolis, EPAGRI, $341 \mathrm{p}$.

Nora, I.; E. R. Hickel \& H. F. Prando. 2000. Moscas-das-frutas nos Estados Brasileiros: Santa Catarina, p. 271-275. In: A. MALAVASI \& R. A. ZuCCHI (edit.). Moscas-das-frutas de importância econômica no Brasil: conhecimento básico e aplicado. Ribeirão Preto, Holos Editora, 327 p.

Orth, A. L.; L. G. Ribeiro \& W. Reis Filho. 1986. Manejo de pragas, p. 341-379. In: EPAGRI (edit). Manual da cultura da macieira. Florianópolis, EPAGRI, $562 \mathrm{p}$.

Puzzi, O. \& A. Orlando. 1965. Estudos sobre a ecologia das "moscas-dasfrutas” (Trypetidae) no Estado de São Paulo, visando o controle racional da praga. Arquivos do Instituto Biológico 32(1): 7-20.

Raga, A.; M. F. de Souza Filho; M. E. Sato \& L. C. Cerávolo. 1996. Dinâmica populacional de moscas-das-frutas em pomares de citros de Presidente Prudente, SP. Arquivos do Instituto Biológico 63(2): 23-38.

SAlLes, L. A. B. 1993a. Efeito da temperatura constante na oviposição e no ciclo de vida de Anastrepha fraterculus (Wied.) (Diptera: Tephritidae). Anais da Sociedade Entomológica do Brasil 22(1): 57-62.

Salles, L. A. B. 1993b. Emergência dos adultos de Anastrepha fraterculus (Wied.) (Diptera: Tephritidae) durante o outono e inverno em Pelotas - RS). Anais da Sociedade Entomológica do Brasil 22(1): 6469.

Salles, L. A. B. 1995. Bioecologia e controle da mosca-das-frutas sul-americana. Pelotas, EMBRAPA - CPACT, 58 p.

SAlles, L. A. B. 1997. A mosca-das-frutas: Biologia, comportamento e controle. Informe Agropecuário 18(189): 62-67.

Salles, L. A. B. 2000. Biologia e ciclo de vida de Anastrepha fraterculus (Wied.), p. 81-86. In: A. Malavasi \& R. A. Zucchi (edit.). Moscasdas-frutas de importância econômica no Brasil: conhecimento básico e aplicado. Ribeirão Preto, Holos Editora, 327 p. 
Salles, L. A. B. \& A. Kovaleski. 1990. Moscas-das-frutas em macieira e pessegueiro no Rio Grande do Sul. Horti Sul 1(3): 5-9.

Salles, L. A. B.; F. L. C. CARvalho \& C. R. Júnior. 1995.Efeito da temperatura e umidade do solo sobre pupas e emergência de Anastrepha fraterculus (Wied.). Anais da Sociedade Entomológica do Brasil 24(1): 147-152.

Suplicy Filho, N.; D. A. Oliveira; E. A. Bitran \& J. Teófilo Sobrinho. 1987. Contribuição ao conhecimento da flutuação populacional das “moscas-das-frutas” em citros. O Biológico 54(1/4): 63-70.

Steyskal, G. C. 1977. Pictorial key to species of the genus Anastrepha (Diptera: Tephritidae). Washington, The Entomological Society of Washington, 35 p.

Taufer, M.; J. C. do Nascimento; I. B. M. da Cruz \& A. K. De Oliveira. 2000. Efeito da temperatura na maturação ovariana e longevidade de Anastrepha fraterculus (Wied.) (Diptera: Tephritidae). Anais da Sociedade Entomológica do Brasil. 29(4): 639-648.

ZahleR, P. M. 1990. Moscas-das-frutas em três pomares do Distrito Federal: Levantamento das espécies. Ciência e Cultura 42(2): 177-182.

Zucchi, R. A. 2000a. Taxonomia. p. 13-24. In: A. Malavasi \& R. A Zucchi (edit.). Moscas-das-frutas de importância econômica no Brasil: conhecimento básico e aplicado. Ribeirão Preto, Holos Editora, 327 p.

Zucchi, R. A. 2000b. Espécies de Anastrepha, sinonímias, plantas hospedeiras e parasitóides. p. 41-48. In: A. Malavasi \& R. A. ZuCCH (edit.). Moscas-das-frutas de importância econômica no Brasil: conhecimento básico e aplicado. Ribeirão Preto, Holos Editora, 327 p. 